MIC90 of gentamicin were $2-16 \mu \mathrm{g} / \mathrm{mL}, 4 \mu \mathrm{g} / \mathrm{mL}$ and $8 \mu \mathrm{g} /$ $\mathrm{mL}$, respectively.

Conclusion In South Korea, the antimicrobial resistance of $N$. gonorrhoeae is very severe and most isolates are multi-drug resistant to penicillin $\mathrm{G}$, tetracycline, and fluoroquinolones. PenA-10.001 and penA-34.001, which are mainly isolated in South Korea, are thought to be the pre-stage of ceftriaxoneresistant germs in Japan and Europe, and the possibility of highly resistant germs is highly increased in South Korea. Enhanced antimicrobial resistance surveillance is necessary to prevent transmission of these strains.

Disclosure No significant relationships.

\section{P005 GONORRHEA IN THE ERA OF AMR, DIAGNOSTIC NEEDS FOR IMPROVED ANTIMICROBIAL STEWARDSHIP IN LOW AND MIDDLE INCOME COUNTRIES}

${ }^{1}$ Cecilia Ferreyra*, ${ }^{2}$ Teodora Wi, ${ }^{3}$ Cassandra Kelly Cirino, ${ }^{1}$ Jennifer Osborn. ${ }^{1}$ FIND, AMR Program, Geneva, Switzerland; ${ }^{2}$ World Health Organization, Department of Reproductive Health and Research, Geneva, Switzerland; ${ }^{3}$ FIND, Emerging Threats, Geneva, Switzerland

\subsection{6/sextrans-2019-sti.217}

Background In 2012, the World Health Organization (WHO) estimated 78 million cases of Neisseria gonorrhoea (NG) worldwide and recent reports indicate an increase in NG resistance to current antibiotic therapies globally. Chlamydia trachomatis (CT) infection is more prevalent than NG and current syndromic management guidelines are designed to simultaneously treat both bacteria with a combination of antibiotics to avoid missing treatment. In resource constrained settings many patients with urethral or vaginal discharge presenting at primary healthcare settings (PHC) are overtreated due to syndromic management, which lacks appropriate diagnostic tools for CT and NG differentiation in these settings.

Methods To address this gap, the Foundation for Innovative New Diagnostics (FIND) and WHO collaborated on the development of Target Product Profiles (TPPs) to guide the development of appropriate diagnostics tools for improved clinical management of NG and CT infections. Using a Delphi-like process involving two serial surveys to solicit input from over 52 experts.

Results Two TPPs for the appropriate identification of NG (and NG/CT) and its susceptibility/resistance profile to antibiotics were developed and will be publicly available on the WHO and FIND websites. A request for proposals is in process to stimulate the development of diagnostic tools that meet the technical characteristics of these TPPs.

Conclusion TPPs for rapid identification of NG-positive patients at PHC and for identification of NG susceptibility/ resistance to antibiotics are urgently needed. Technology advancements over the past years may enable development of improved tests to support uptake and wide scale use in PHC. A reflex antibiotic susceptibility test would be useful to further guide prescription of current therapies. Results of this work will guide the development of new and appropriate diagnostics in the next 3-5 years, to enable improved patient management and conservation of new antibiotics that will become available.

Disclosure No significant relationships. OF COUPLE'S HIV TESTING IN ETHIOPIA

${ }^{1}$ Tewodros Getachew Hailemariam*, ${ }^{\text {P}}$ Patrick Rawstorne, ${ }^{2}$ Mitike Sisay, ${ }^{1}$ Sally Nathan. ${ }^{1}$ The University of New South Wales, School of Public Health and Community Medicine, Kensington, Australia; ${ }^{2}$ Addis Ababa University, School of Public Health, Addis Ababa, Ethiopia

\subsection{6/sextrans-2019-sti.218}

Background Some evidence indicates that Couple HIV Testing \& Counselling (CHTC) is an approach that could be used to enable more people to be reached. However, little is known about how couples may use this service and what their concerns are around the approach. This study aimed to understand how individuals who had ever been in a long-term heterosexual relationship intended to use CHTC in Ethiopia and their beliefs about its benefits and potential harms.

Methods A qualitative study was conducted in Addis Ababa, Ethiopia. We interviewed individuals who had ever been in a long-term heterosexual relationship $(n=21)$, and key-informants $(n=11)$ including religious leaders, health-care providers and case managers. The interview data were transcribed verbatim and thematically analysed using NVivo 11 . The data were coded to look for concepts and patterns across the interviews, and relevant themes and sub-themes identified which captured key aspects related to individual's views on undertaking HIVtesting with a sexual partner.

Results Most participants regarded CHTC as an important HIV-testing approach for people who are in a long-term heterosexual relationship and expressed the view that there was 'nothing like testing together'. However, many participants said they would prefer to be tested alone to check their own HIV-status before undertaking or deciding to undertake CHTC - 'first alone then together'. This strategy was expressed as a way of managing their concerns about being HIV-positive and 'fear of the consequences' including potential accusations of infidelity, relationship break-up, and potentially exposed in the community as being HIV-positive.

Conclusion The findings of this study suggest that while CHTC has been actively promoted in Ethiopia, people may be concerned about undertaking CHTC without prior individual HIV-testing and have developed ways of making the process work for them. More work is needed to understand the approach couples considering is a more dignified, effective and cost-effective way.

Disclosure No significant relationships.

\section{P007 UPTAKE OF COUPLES HIV TESTING AND COUNSELLING IN SUB-SAHARAN AFRICA: A SYSTEMATIC REVIEW AND META-ANALYSIS}

${ }^{1}$ Tewodros Getachew Hailemariam*, ${ }^{1}$ Sally Nathan, ${ }^{2}$ Canaan Seifu, ${ }^{1}$ Patrick Rawstorne. ${ }^{1}$ The University of New South Wales, School of Public Health and Community Medicine, Kensington, Australia; ' Wolaita Sodo University, School of Public Health, Wolaita Sodo, Ethiopia

\subsection{6/sextrans-2019-sti.219}

Background This systematic review and meta-analysis aimed to estimate and characterise the uptake of Couples HIV Testing and Counselling (CHTC) in Sub-Saharan African countries. 